\title{
Rendimiento escolar en la asignatura de Lenguaje y Comunicación en el nivel Secundario de Bolivia
}

\author{
School performance in the subject of Language and Communication at the Secondary \\ level of Bolivia \\ Desempenho escolar na disciplina de Língua e Comunicação no nível médio da Bolívia
}

ARTÍCULO DE INVESTIGACIÓN

\author{
Araceli Santos Bacallao \\ araceli056@hotmail.com \\ ORCID: 0000-0002-8778-9889
}

ALSIE “Consultores Pedagógicos” SRL Universidad Autónoma del Beni “JOSÉ BALLIVIÁN" Bolivia

Recibido 09 de diciembre 2020 | Arbitrado y aceptado en 26 de diciembre | Publicado en diciembre 2020

\section{RESUMEN}

Se realizó un estudio para determinar en los estudiantes el rendimiento académico en el área de Lenguaje y comunicación en las escuelas secundaria en Bolivia. Teniendo como propósito elaborar una propuesta de un nuevo diseño curricular dirigido al área de Lenguaje y Comunicación para el nivel secundario, en el segundo y tercer ciclo en las Unidades Educativas de Cochabamba. La metodología aplicada en la investigación respondió al enfoque dialéctico que sirvió de soporte a los métodos holístico, sistémico y holográfico. La recolección de información fue mediante la técnica de entrevista, encuesta y plantillas de calificaciones. Por lo que evidenció que existen bajas calificaciones de Secundaria en la asignatura, estas se encontraban ubicadas entre 36 y 45 puntos. Se consideró que para ser el grado terminal de Secundaria estos resultados no correspondían con los que se perseguían y basado previamente en el diagnóstico, se elaboró una propuesta curricular en la asignatura objeto de estudio.

Palabras clave: Diseño Curricular; lenguaje y comunicación, enseñanza y aprendizaje, nivel secundario, rendimiento académico

\begin{abstract}
A study was conducted to determine students' academic performance in the area of Language and Communication in secondary schools in Bolivia. With the purpose of elaborating a proposal for a new curricular design directed to the area of Language and Communication for the secondary level, in the second and third cycle in the Educational Units of Cochabamba. The methodology applied in the research responded to the dialectical approach that served as support for the holistic, systemic and holographic methods. The information was collected using the technique of interview, survey and rating templates. Therefore, it showed that there are low Secondary grades in the subject; these were located between 36 and 45 points. It was considered that to be the terminal grade of secondary these results did not correspond to those that were pursued and based previously on the diagnosis; a curricular proposal was elaborated in the subject under study.
\end{abstract}

Key words: Curricular Design; language and communication, teaching and learning, secondary level, academic achievement 


\section{RESUMO}

Foi realizado um estudo para determinar o desempenho acadêmico dos alunos na área de Linguagem e Comunicação em escolas de ensino médio na Bolívia. Com o objetivo de elaborar uma proposta de novo desenho curricular dirigido à área de Linguagem e Comunicação para o nível médio, no segundo e terceiro ciclos nas Unidades Educacionais de Cochabamba. A metodologia aplicada na pesquisa respondeu à abordagem dialética que serviu de suporte para os métodos holístico, sistêmico e holográfico. A informação foi recolhida através da técnica de entrevista, inquérito e modelos de classificação. Portanto, evidenciou que há baixas notas do ensino médio na disciplina, situando-se entre 36 e 45 pontos. Considerou-se que por ser o grau terminal do Ensino Médio esses resultados não correspondiam aos que foram buscados e com base anteriormente no diagnóstico, foi elaborada uma proposta curricular na disciplina em estudo.

Palavras-chave: Curricular Design; linguagem e comunicação, ensino e aprendizagem, nível médio, desempenho acadêmico

\section{INTRODUCCIÓN}

En cada país e instituciones educativas dentro de los proyectos formativos están los Diseños curriculares, teoría que hace énfasis en el contenido de la cultura que se traslada a la escuela, como proceso que mediatiza el mundo de la vida con el mundo de la escuela. La relación fundamental que atiende lo curricular es el vínculo entre el objeto y el contenido, como expresión pedagógica de la cultura y tiene como centro la dimensión desarrolladora, lo que asegura una concepción eficaz del proceso de Enseñanza Aprendizaje. Cada contexto es diferente y de acuerdo con las necesidades, demandas sociales en relación con la formación de los recursos humanos, el desarrollo económico-social, así como los conocimientos científicos y técnicos, es que se elaboran. Ejemplos: en Argentina el estudiante construye el conocimiento solo, no se evidencia la dinámica y dialéctica del proceso de enseñanza.
Otro aspecto importante es que todo el contenido es práctico por lo tanto lo metodológico, las habilidades, capacidades y competencias no han sido tomadas en cuenta para el desarrollo futuro de ese hombre en la sociedad, según Acosta, (2012). El Diseño Curricular aplicado en Chile tiene como objetivo fundamental la investigación, interpretación y producción de obras literarias, el enriquecimiento de su competencia lectora, para que el aprendizaje sea significativo. (Ministerio de Educación Chile, 2009). En Bolivia, en los momentos históricos sociales que vive el país en condiciones tan complejas, identificadas por la diversidad cultural, se requiere que la escuela forme ciudadanos con una solidez significativa, tanto en sus rasgos éticos como intelectuales, profesionales, competitivos que desarrollen las competencias $\mathrm{y}$ que sean portadores de los valores, sentimientos y convicciones, en consonancia con lo estratégico para la nación y el país y lo táctico, dirigido a los docentes de base, área y el núcleo de escuelas. Estos currículos deben identificarse por la flexibilidad y apertura, de acuerdo con el contexto social y comunitario. Es indispensable trasladar a la escuela los contenidos propios de la profesión, de forma tal que el currículo garantice la formación de los estudiantes y a la vez responda a las necesidades sociales. De igual forma es preciso sistematizar, articular los contenidos (conocimientos, habilidades, capacidades y valores), planificarlos y seleccionarlos correctamente por su incidencia en la formación de los estudiantes y en su rendimiento académico.

El problema identificado en la investigación es el bajo rendimiento académico en el área de Lenguaje y Comunicación del nivel secundario en las Unidades Educativas (UE) de Cochabamba, de las que se estudian y caracterizan cinco. 
El diseño estudiado no cumple con las dimensiones y grado de profundidad, con la lógica coherente de los contenidos para el grado y a la vez incide en los resultados que alcanzan los estudiantes. Otro aspecto a considerar es la selección de la bibliografía con la que se trabaja, que independientemente de no ser la misma para todo el país en la asignatura, no incorpora contenidos que respondan a objetivos representativos y significativos dentro de la cultura universal y latinoamericana, además de no obedecer a una organización lógica. La educación en el departamento se rige por la ley 070 de la Reforma Educativa desde 2010 que entre los propósitos principales, planteó el proceso educativo a partir del desarrollo de la interculturalidad, el bilingüismo, y la participación popular con un enfoque político, económico y social (Reforma Educativa, 2012) para crear las bases teórico-prácticas e investigativas y establecer las normas del nuevo modelo educativo de carácter sociocomunitario y productivo, que surge como respuesta al cambio político que vive el país.

La parte de la realidad estudiada, el contexto, es la ciudad de Cochabamba ubicada en el corazón de Bolivia. Está rodeada por seis departamentos con los cuales comparte sus variados climas, culturas, pisos ecológicos y recursos naturales. La población estudiantil es variada, de distintas escalas sociales que coexisten en las unidades educativas donde el trabajo está dirigido a contribuir a la formación $\mathrm{y}$ fortalecimiento de las identidades culturales y lingüísticas a partir de los conocimientos y saberes de las culturas indígenas originarias y sectores urbanos populares.

El propósito de la investigación es elaborar un nuevo Diseño curricular dirigido al área de Lenguaje y Comunicación para el nivel secundario, en el segundo y tercer ciclo en las Unidades Educativas de Cochabamba. El nuevo diseño que se propone se caracteriza por: dosificar los contenidos de forma progresiva con un enfoque humanista personalizado que permitirá atender las diferencias individuales en el aprendizaje, tributar al desarrollo de una cultura general integral y elevar el nivel de conocimiento y formar habilidades, capacidades y competencias comunicativas, para que, al terminar el nivel secundario, el estudiante posea un perfil que le permita ingresar a la Educación Superior.

Lo expuesto permite mantener una visión basada en la importancia de la mejora en la educación en el área de Lenguaje y Comunicación, necesaria para transformación e incidencia positiva en el rendimiento académico de los estudiantes, teniendo en cuenta el enfoque humanista personalizado y en de cultura general integral.

\section{MÉTODO}

La metodología aplicada en la investigación responde al enfoque dialéctico que sirve de soporte a los métodos: holístico, sistémico y holográfico, es decir complejo, los que están presentes en todo el proceso de investigación científica y que contribuyeron a la demostración del problema que presentan los estudiantes en la asignatura Lenguaje y Comunicación de forma lógica y seleccionando de cada parte de la estructura investigativa sus componentes que están en unidad y contradicción, concebidos como un todo, ya que en la solución de estas se cumple el objetivo de la investigación. (Álvarez, 2016). Cada parte del proceso de investigación se realizó partiendo del problema, tomando como referencia la teoría del diseño curricular y su relación con el proceso formativo. También se aplica el método de la observación científica y la medición, donde se construyeron tablas estadísticas para cuantificar las calificaciones alcanzadas por los grupos de estudiantes que constituyen la muestra (prueba diagnóstica) las que 
evidenciaron la necesidad de estudiar. Además, se aplicaron las siguientes técnicas: una encuesta, entrevistas y la revisión documental.

Al personal docente, como componente activo del proceso de enseñanza y aprendizaje se le aplicó la entrevista para obtener información sobre el comportamiento del rendimiento académico de los estudiantes, la metodología aplicada en el aula y el grado de participación de los estudiantes en el proceso de aprendizaje. También se aplicó la entrevista a una funcionaria de la dirección de educación para conocer sus criterios sobre proyectos educativos y los diseños curriculares vigentes en la asignatura Lenguaje y Comunicación. Los métodos y técnicas aplicadas fueron las señalas en la Tabla 1.

Tabla 1. Instrumentos y técnicas en la recolección de datos

\begin{tabular}{lll} 
Grupo de muestra & Instrumento & Técnica \\
Unidades Educativas & Registro de calificaciones & Revisión de calificaciones \\
Profesores & Guía de entrevista a profesores & Entrevista \\
Directores Distrito & Cuestionario & Encuesta \\
Funcionaria & Guía de entrevista & Entrevista \\
\hline
\end{tabular}

Cuatro instrumentos fueron aplicados para obtener información sobre los aspectos positivos y negativos que influyen en la problemática: revisión de calificaciones, encuesta y 2 entrevistas. Los instrumentos aplicados fueron reveladores de la situación real que presentaban los estudiantes relacionados con las causas del bajo rendimiento académico. Los resultados de la aplicación fueron procesados en cuadros y gráficos estadísticos con sus correspondientes análisis donde se evidencia en porcentaje los resultados obtenidos.

Los Procedimientos aplicados en el proceso de investigación fueron:

- Revisión de calificaciones. se realizó el análisis basado en los resultados académicos mediante la aplicación de una prueba diagnóstica para medir el grado de conocimiento de los estudiantes.
- Las calificaciones fueron las siguientes. En la escala de 1 a 35 puntos (reprobados), 36 a 45 (bajo) y 46 a 55 (regular).

- Entrevista a profesores. se aplicaron preguntas cerradas acerca del rendimiento académico, las metodologías aplicadas y la atención personalizada.

- Encuesta a los directores. referidas, como ya se destacó, a los proyectos educativos y su relación con el currículo.

- La población seleccionada para este estudio. se correspondió con el grado de participación en el proceso educativo: 10 Docentes de Lenguaje y Comunicación, 10 Unidades Educativas, 25 Directores Distritales, 1 funcionaria de la Dirección Departamental de Educación y se tuvo como referente el registro de calificaciones de 5 Unidades Educativas equivalentes a un total de 306 estudiantes. 
RESULTADOS

Los resultados que se muestran están en correspondencia con la aplicación de las técnicas de recolección de datos. Seguidamente se exponen los resultados más relevantes que demuestran el problema declarado. A continuación en la Tabla 2 muestra el resumen de los resultados en las calificaciones de estudiantes de secundaria en la asignatura de Lenguaje y Comunicación de 5 UE de Cercado 1.

Tabla 2. Calificaciones del total de los estudiantes de secundaria en la materia de lenguaje y comunicación de 5 unidades educativas

\begin{tabular}{ccccccccc} 
Calificación & \multicolumn{4}{c}{ Cantidad de estudiantes por unidad educativa } & \multicolumn{2}{c}{ Total } \\
& $\begin{array}{c}\text { Unidad } \\
\text { Educativa 1 } \\
\text { 9no }\end{array}$ & $\begin{array}{c}\text { Unidad } \\
\text { Educativa 2 } \\
\text { 9no }\end{array}$ & $\begin{array}{c}\text { Unidad } \\
\text { Educativa 3 } \\
\mathbf{1 0 m 0}\end{array}$ & $\begin{array}{c}\text { Unidad } \\
\text { Educativa 4 } \\
\mathbf{1 1 n o}\end{array}$ & $\begin{array}{c}\text { Unidad } \\
\text { Educativa 5 } \\
\text { 12vo }\end{array}$ & Cant & Porc \\
\hline $\mathbf{1 - - 3 5}$ & 6 & 23 & 12 & 35 & 25 & 101 & $33 \%$ \\
$\mathbf{3 6 - - 4 5}$ & 9 & 33 & 16 & 44 & 38 & 140 & $46 \%$ \\
$\mathbf{4 6 - - 5 5}$ & 2 & 4 & 1 & 3 & 10 & 20 & $7 \%$ \\
$\mathbf{5 6 - - 6 5}$ & 1 & 7 & 1 & 3 & 3 & 15 & $5 \%$ \\
$\mathbf{6 6 - - 7 0}$ & & 6 & & & 1 & 7 & $2 \%$ \\
Abandonan & 1 & 6 & & 11 & 5 & 23 & $8 \%$ \\
Total & 19 & 79 & 30 & 96 & 82 & 306 & $100 \%$ \\
\hline
\end{tabular}

Nota: Actas de calificaciones de las unidades educativas, 2018.

En cuanto a las calificaciones el 33\% reprobó la asignatura Lenguaje y comunicación; el $46 \%$, aunque haya aprobado, lo hizo con el mínimo de nota, mostrando un bajo rendimiento académico, además de que hubo un $7 \%$ de estudiantes con notas regulares entre 46 y 55 . Solamente el 5\% alcanza resultados satisfactorios y el 2\% alcanza notas excelentes.

Resultado de la entrevista a Directores Distritales (Gráfico 1) se muestra los datos obtenidos a través de la pregunta ¿Existen diseños curriculares emitidos por DDE para el nivel secundario?

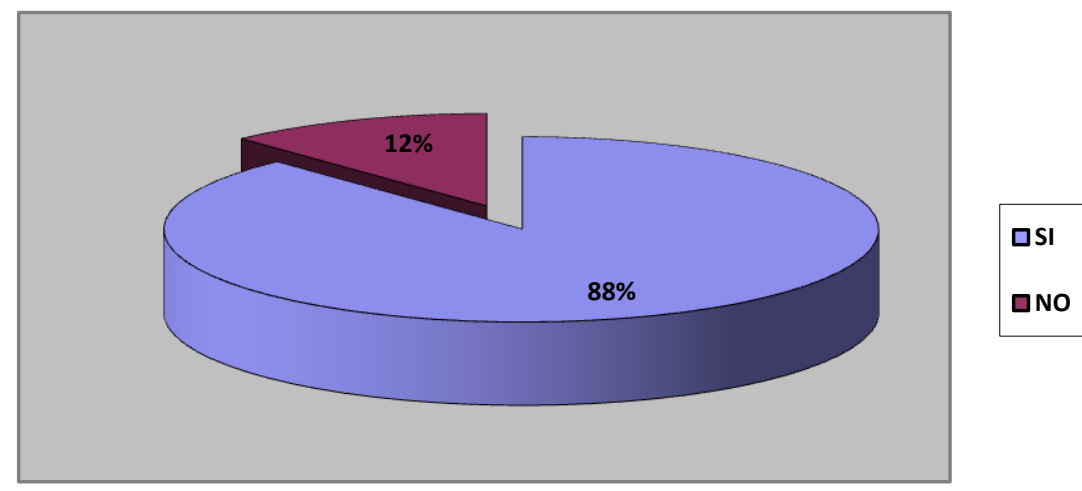

Gráfico 1. Existencia de diseños curriculares emitidos por SEDUCA 
De acuerdo con el gráfico 1, el $88 \%$ de los directores distritales considera que sí existen diseños curriculares emitidos por DDE para el nivel secundario. Por tanto, esto sugiere que se trabaja en Cochabamba bajo modelos establecidos para todos los estudiantes, es decir, desde el nivel macrocurricular se diseña el currículo. Independientemente de ello, al llegar a las Unidades Educativas, en el nivel micro, no se mantiene la unidad de criterios, a lo que se añade la heterogeneidad en lo referido a la bibliografía con la que se trabajó.

Seguidamente, se presenta los datos obtenidos a partir de la pregunta "De acuerdo con su experiencia, ¿cuántos proyectos educativos del nivel secundario c onoce? (Tabla 3).

Tabla 3. Directores distritales según conocimiento de proyectos educativos de nivel secundario y su relación con el currículo

\begin{tabular}{|c|cc|}
\hline Cantidad de proyectos conocidos & Respuestas de Directores & $\%$ \\
\hline 1 a 2 & 18 & 72 \\
\hline 3 a 4 & 5 & 20 \\
\hline 5 a 6 & - & - \\
\hline Más de 6 & 2 & 8 \\
\hline Ninguno & - & - \\
\hline Total & 25 & 100 \\
\hline
\end{tabular}

Nota: Encuesta a directores distritales, 2018

Según los datos presentes en la Tabla 3, el $72 \%$ de los 25 directores distritales encuestados, indicó que conoce entre 1 a 2 proyectos educativos para el nivel secundario y que se correspondían con el diseño curricular. Otros cinco directores dijeron conocer entre 3 a 4 proyectos y solamente 2 señalaron que conocen más de 6 proyectos que tienen en cuenta el diseño curricular. Por lo tanto, los directores tienen conocimiento sobre proyectos educativos, aunque plantean conocer pocos proyectos de este tipo, aplicados al nivel secundario.

Con relación a la interrogante acerca del nivel de aprendizaje de los estudiantes de bachiller en Cochabamba. En el gráfico 2 se muestran los resultados, quedando demostrado que el $48 \%$ declara que los estudiantes de secundaria básica de Cochabamba al concluir los estudios presentan un nivel regular en lo referido a su aprendizaje, mientras que el $28 \%$ lo califica como malo. 


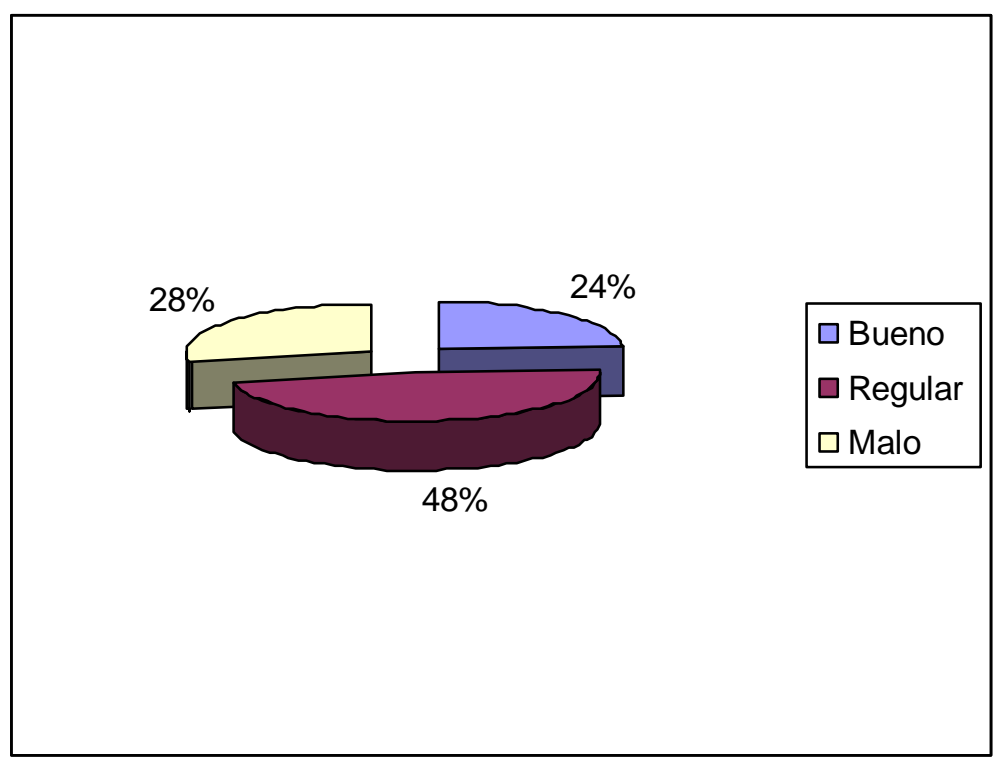

Gráfico 2. El nivel de aprendizaje de los estudiantes de bachiller en Cochabamba. (Fuente: Encuesta a directores distritales, 2018)

\section{Resultado de entrevista a los docentes de Lenguaje y comunicación}

Al respecto acerca de Pregunta: El alumno al terminar el grado académico tiene dominio de: ¿Ortografía, redacción, interpretación e investigación de textos orales y escritos? Los entrevistados manifestaron (Gráfico 3), que el $80 \%$ de los estudiantes cuando terminan el nivel secundario presentan dificultades en la producción de textos orales y escritos.

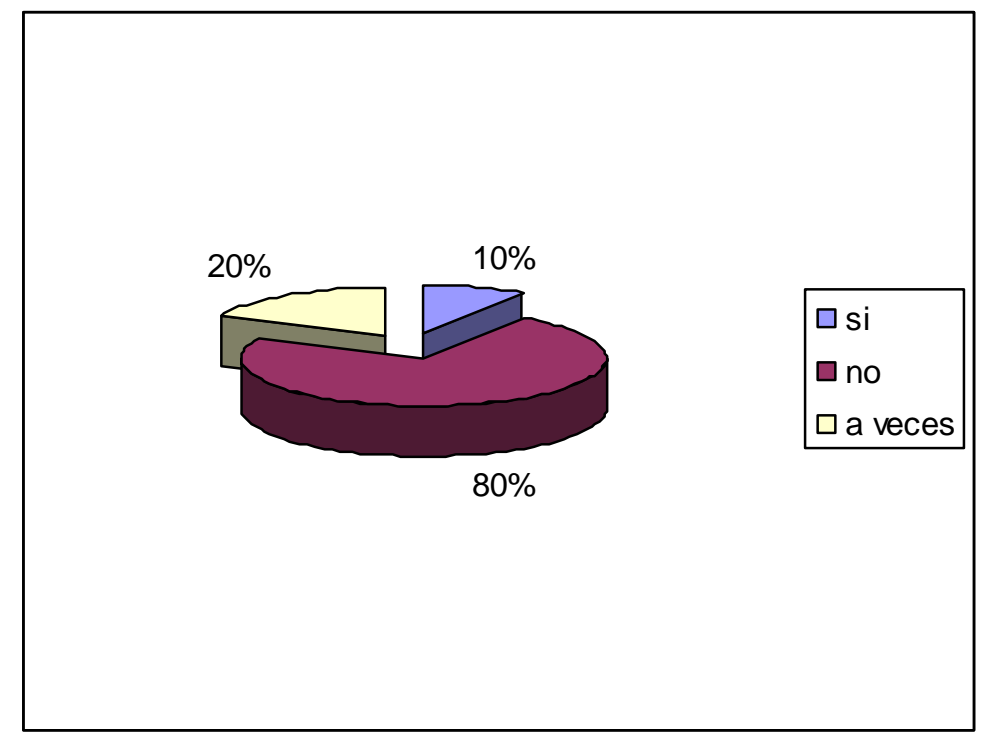

Grafico 3. Dominio de Ortografía, redacción, interpretación e investigación de textos orales y escritos (Fuente: Guía de entrevista a docentes, 2018) 
Como conclusión de las entrevistas aplicadas a profesores del nivel secundario se constató que deben revisarse los programas de la asignatura del Lenguaje y comunicación, reconsiderar la organización, unidad y selección de los contenidos, conjuntamente con la bibliografía que se indica, con el objetivo de lograr un mejor rendimiento académico en la asignatura Lenguaje y comunicación.

Además, al realizar la triangulación entre las técnicas aplicadas, se evidenció la necesidad de reconsiderar el Diseño Curricular en la asignatura ya mencionada, sobre la base de los resultados alcanzados por los estudiantes y los criterios emitidos por las autoridades educativas.

Los datos mostrados anteriormente demuestran que a partir de las respuestas de los docentes, no se brinda una atención personalizada a los estudiantes, principalmente debido a la excesiva cantidad de alumnos por aula y a elementos de carácter metodológico que también incide en el problema declarado. Esto influye en su aprendizaje puesto que tienen dificultades en la ortografía, redacción, interpretación de textos orales y escritos.

Por lo antes expuesto, resulta necesario estudiar el currículo, los contenidos, organización, así como su pertinencia y la bibliografía indicada, de forma tal que se logre una relación lógica entre estos. Todos los elementos relacionados incidieron en que los estudiantes alcancen un proceso formativo coherente y sistemático, que favorezca la adquisición de conocimientos, el desarrollo de habilidades, competencias y en consecuencia un rendimiento académico favorable.

Para finalizar, en el ítem (Gráfico 4) acerca de los contenidos que satisfacen las necesidades educativas de los estudiantes. Queda demostrado que el $80 \%$ de los profesores plantean que los contenidos que se imparten actualmente en la asignatura de Lenguaje no satisfacen las necesidades educativas de los estudiantes, existiendo carencias en las estructuras gramaticales y la redacción de diferentes textos párrafos y composiciones.
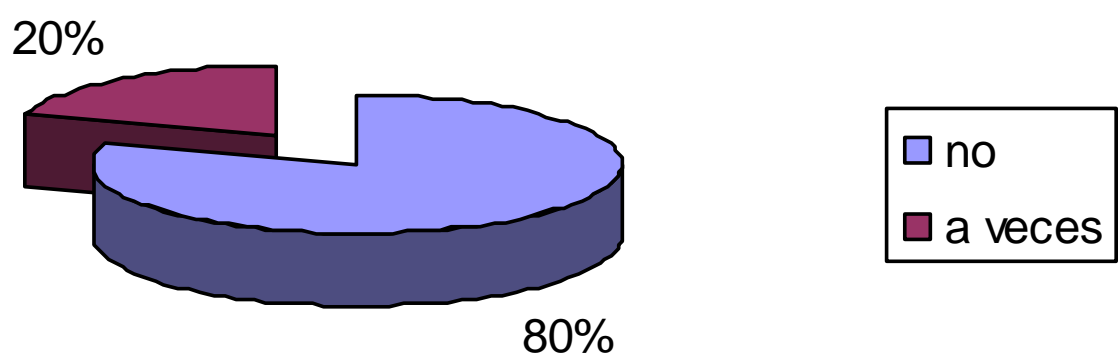

$80 \%$

Gráfico 4. Contenidos que satisfacen las necesidades educativas de los estudiantes. (Fuente: Guía de entrevista a docentes, 2018). 
Tabla 4. Análisis de los resultados finales de los instrumentos aplicados

\section{Resumen de los resultados de las entrevistas a docentes}

El $80 \%$ plantea que los contenidos que se imparten actualmente en la asignatura de lenguaje, no satisfacen las necesidades educativas de los estudiantes

El $70 \%$ imparte sus clases por un libro de texto. Quiere decir que ellos se preparan según el texto y con diferentes editoriales

El 90 \% respondió que no brinda atención personalizada

El $80 \%$ de los estudiantes cuando terminan el nivel secundario presentan dificultades en la ortografía, producción e interpretación de textos orales y escritos

El 90 \% manifiesta tener más de 40 estudiantes en su sala de clases
Resumen de los resultados de las encuestas a directores

El 72 \% indicó que conocen entre 1 y 2 proyectos educativos que tienen en cuenta el currículo.
El $23 \%$ no tiene conocimiento de proyectos educativos personalizados en correspondencia con el currículo.
El $48 \%$ considera que los estudiantes de secundaria básica al concluir los estudios, presentan bajo nivel académico.

Resultado de la entrevista a funcionaria de DDE

Solo conoce una UE con proyecto educativo personalizado
En las UE funcionan con $30-40$ alumnos por curso

\begin{abstract}
La mayoría de los centros no tienen condiciones de infraestructura y personal preparado
\end{abstract}

La situación económica y social de la población

\section{Hallazgos}

La mayoría de los estudiantes tienen calificaciones por debajo de (36 y 45) puntos con 46\% y un 33\%(1 a 35) de reprobación, bajo dominio de la ortografía, redacción, interpretación e investigación de textos orales y escritos, según la percepción del $80 \%$ de los profesores de lenguaje y comunicación.
Se presenta gran cantidad de estudiantes por aula (muchas veces con más de 40 adolescentes en cada curso), lo que dificulta brindar una atención personalizada a los estudiantes y hasta el momento no existe un currículum nacional actualizado en el área y a nivel secundario con una bibliografía única para todo el país. Además, al derivarse hasta el microcurrículo no hay unidad de criterios. 


\section{Propuesta del diseño curricular para la asignatura lenguaje y comunicación a nivel secundario}

El proceso de la investigación está basado en la teoría de los procesos conscientes de Álvarez (2016), que propone una lógica para la solución de problemas de la vida en el estudiante a partir de las dimensiones del proceso de diseño curricular. (p. 127).

Se propone rediseñar la asignatura en el nivel secundario con una propuesta de Diseño Curricular en el área de Lenguaje y Comunicación desde una lógica de contenidos que lleve al estudiante a la comprensión e interpretación de textos literarios en el nivel secundario, también es esencial su aplicación personalizada para llevar a las instituciones educativas de su estado actual a un estado aspirado, alcanzar un mejor rendimiento académico y formar al nuevo hombre boliviano multifacéticamente.

\section{Fundamentación teórica}

Se estudiaron las categorías conceptuales relacionadas con el objeto y campo de acción de la investigación, sobre la base de algunos de los autores a los cuales se adscribe la investigadora.

Currículo. "Es un mediador entre el proyecto histórico-cultural de una sociedad y el proyecto formativo de una institución docente". (Álvarez, (2019, p. 127). El diseño es el primer paso de todo proceso formativo, donde se traza el modelo a seguir y se proyecta: la planificación y organización del proceso de enseñanza aprendizaje (PEA) con sus dimensiones y componentes.

Rendimiento académico. Figueroa (2004), expresa en su obra: Sistemas de Evaluación Académica, define el rendimiento académico como: "El producto de asimilación del contenido de los programas de estudio, expresado en calificaciones dentro de una escala convencional, como una medida de capacidades del estudiante, expresando lo que éste ha aprendido a lo largo del proceso". Asimismo, el referido autor define el rendimiento académico de modo general como: "El conjunto de transformaciones esperadas en el estudiante a través del proceso enseñanza aprendizaje, que se manifiesta mediante el crecimiento y enriquecimiento de la personalidad en formación". (p.25).

Lenguaje y Comunicación. Es la forma ordenada, clara, coordinada de expresar y comprender en forma oral y escrita. La palabra da vida a los seres, a las cosas y da sentido, significado y valor a los conceptos, teorías e ideas, hace al hombre un ser sociable, (Gómez, 2000). Además, se plantea que esta área del saber posibilita crear estructuras gramaticales que reflejan las intenciones comunicativas para las relaciones humanas.

Educación personalizada: proceso formativo que toma en cuenta a las dimensiones de la persona, considerándola como un ser único e irrepetible a los demás. (Soacha, 2001). Es de gran importancia en la formación de valores, sentimientos, convicciones y normas de conducta como parte de la personalidad de los estudiantes.

\section{Objetivo de la propuesta}

El objetivo como aspiración y propósito de la transformación del objeto de investigación a un estado deseado debe de reflejar cómo resolver el problema o contribuir a su solución:

Seleccionar, organizar y sistematizar los contenidos del programa Lenguaje y Comunicación de forma pertinente y teniendo como sustento las relaciones entre los componentes desde un enfoque humanista personalizado, que permitirá atender las 
diferencias individuales en el aprendizaje, tributar a desarrollo de una cultura general integral y mejorar el rendimiento académico, para formar al nuevo hombre boliviano multifacéticamente.

\section{Planificación del Diseño}

Se realiza una presentación de los aspectos que formarán parte del diseño de la asignatura de Lenguaje y Comunicación en el nivel secundario.

- Problema. Influencia de las diferentes etapas de la historia de la literatura en la cultura.
- Objeto. Contenidos de Lenguaje y Comunicación

- Objetivo. Caracterizar y comparar géneros, movimientos y estilos literarios para manifestarse culturalmente en su medio.

A continuación, se presenta la planificación curricular del área de lenguaje y comunicación del nivel secundario según ciclos y grados.

\section{Segundo ciclo del nivel secundario}

\section{COMPETENCIA DEL CICLO}

- El estudiante valora obras y géneros literarios bolivianos, universales y produce textos basados en ellos.

\section{CONTENIDOS}

- 9no de secundaria (3)

- Historia de la literatura boliviana $10 \mathrm{mo}$ de secundaria (4)

- Historia de la literatura universal

\section{Tercer ciclo del nivel secundario}

\section{COMPETENCIA DEL CICLO}

- Analiza y produce textos basados en el pensamiento literario latinoamericano y contemporáneo

\section{CONTENIDOS}

- 11no de secundaria (5)

- Manifestaciones del pensamiento literario latinoamericano.

- 12vo de secundaria (6)

- Literatura contemporánea 


\section{Ejemplo comparativo del diseño actual de 9no grado y nuevo diseño propuesto}

\begin{tabular}{|c|c|c|c|c|c|c|c|c|}
\hline \multirow[t]{6}{*}{$\begin{array}{l}\text { Curso } \\
\text { (9) } \\
\text { Medio }\end{array}$} & $\begin{array}{l}\text { UT } 1 \\
\text { La orator }\end{array}$ & & $\begin{array}{l}\text { UT2Producc } \\
\text { textos }\end{array}$ & $\begin{array}{l}\text { Un de UT } 3 \\
\text { Historia de la } \\
\text { Universal }\end{array}$ & Lit. & $\begin{array}{l}\text { UT } 4 \text { Leng } \\
\text { literario }\end{array}$ & $\begin{array}{c}\text { uaje UT } 5 \text { Anális } \\
\text { literario }\end{array}$ & $\begin{array}{l}\text { sis UT } 6 \\
\text { Mediosde } \\
\text { comunicación }\end{array}$ \\
\hline & Contenidos & & Contenidos & Contenidos & & Contenidos & Contenidos & Contenidos \\
\hline & $\begin{array}{l}\text { Objetivos d } \\
\text { oratoria. } \\
\text { Elementos } \\
\text { externos. } \\
\text { El diálogo. } \\
\text { El discurso }\end{array}$ & & $\begin{array}{l}\text { Tipos. } \\
\text { Operativos. } \\
\text { Explicativos } \\
\text { Didácticos } \\
\text { Informativos } \\
\text { Gramática }\end{array}$ & $\begin{array}{l}\text { Literatura } \\
\text { Griega } \\
\text { Latina } \\
\text { Antiguas } \\
\text { Edad media } \\
\text { Latinoamericana }\end{array}$ & & $\begin{array}{l}\text { Géneros } \\
\text { Lírico } \\
\text { Narrativo } \\
\text { Épico }\end{array}$ & $\begin{array}{l}\text { Concepto } \\
\text { Importancia } \\
\text { Modelo de } \\
\text { análisis } \\
\text { Teoría y } \\
\text { práctica }\end{array}$ & $\begin{array}{l}\text { Género radiofónico } \\
\text { Características } \\
\text { sociales de la } \\
\text { radio. } \\
\text { Prensa } \\
\text { Cronología } \\
\text { Boliviana }\end{array}$ \\
\hline & $\begin{array}{l}\text { UT } 1 \\
\text { Lit Prehispánica }\end{array}$ & & $\begin{array}{l}\text { Lit de la } \\
\text { nia }\end{array}$ & $\begin{array}{l}\text { UT } 3 \\
\text { Lit. Boliviana. EI } \\
\text { Romanticismo }\end{array}$ & $\begin{array}{l}\text { UT } \\
\text { Boli } \\
\text { Bar }\end{array}$ & $\begin{array}{l}4 \text { Lit. } \\
\text { viana. } \\
\text { roco }\end{array}$ & $\begin{array}{l}\text { UT } 5 \text { Lit } \\
\text { Boliviana. } \\
\text { Neoclasicismo }\end{array}$ & $\begin{array}{l}\text { UT } 6 \\
\text { Lit Boliviana. } \\
\text { Realismo }\end{array}$ \\
\hline & Contenidos & Cor & tenidos & Contenidos & Con & tenidos & Contenidos & Contenidos \\
\hline & $\begin{array}{l}\text { La lírica. } \\
\text { Elegía o wanka } \\
\text { Aramays. } \\
\text { Himnos o jaillis. } \\
\text { Tragedia } \\
\text {-Ollantay. } \\
\text { Gramática. } \\
\text {-Composición y } \\
\text { poesía }\end{array}$ & $\begin{array}{l}\text { Lite } \\
\text { de } \\
\text { Las } \\
\text { crór } \\
\text { de } \\
\text { Cab } \\
\text { hida }\end{array}$ & $\begin{array}{l}\text { ratura Boliviana } \\
\text { colonia. } \\
\text { crónicas . .(La } \\
\text { ica "Naufragios" } \\
\text { Ivar Núñez } \\
\text { eza de Vaca, } \\
\text { go) }\end{array}$ & $\begin{array}{l}\text { Causas de su } \\
\text { aparición. } \\
\text { Características en } \\
\text { Bolivia. } \\
\text { Representantes. } \\
\text { Cuento :Adela } \\
\text {.Zamudio. } \\
\text { Lírica:Natalier } \\
\text { Aguirre(Juan de la } \\
\text { Rosa). }\end{array}$ & $\begin{array}{l}\text { Car } \\
\text { Obr } \\
\text { Rep } \\
\text { Juar } \\
\text { Cruz } \\
\text { Gra } \\
\text {-Ve } \\
\text { reg } \\
\text { irreg }\end{array}$ & $\begin{array}{l}\text { cterísticas. } \\
\text { as. } \\
\text { resentantes. } \\
\text { de Santa } \\
\text { nática. } \\
\text { bos } \\
\text { dlares e } \\
\text { ulares. }\end{array}$ & $\begin{array}{l}\text { Concepto. } \\
\text { Características. } \\
\text { Autores. } \\
\quad \text { Bartolomé } \\
\text { Arzans de Urzúa } \\
\text { y Vela } 1676 \text { - } \\
\qquad 1736 \\
\text { Obra :La villa } \\
\text { imperial de } \\
\text { Potosí }\end{array}$ & $\begin{array}{l}\text { Características. } \\
\text { Autores y obras. } \\
\text { Alcides } \\
\text { Arguedas.(Raza de } \\
\text { Bronce). } \\
\text { Armando } \\
\text { Chirveche } \\
\text { El cuento en el } \\
\text { Realismo y la } \\
\text { Guerra del Chaco. }\end{array}$ \\
\hline
\end{tabular}

Fuente: Español y literatura,Cuba-2001 y La Hoguera Bolivia-2008

En el ejemplo anterior se realiza un análisis comparativo donde se reflejan los contenidos existentes en el nivel de 9no grado. Se resaltan los que son innecesarios y los que están muy cargados para que formen parte de una unidad temática en el caso de la UT-3.

En el diseño propuesto se observa de una forma progresiva que los contenidos referidos a las etapas de la literatura boliviana y los autores más significativos e importancia de sus obras, más los contenidos gramaticales, deben incorporarse, como consecuencia del estudio realizado y para lograr alcanzar el estado deseado.

\section{Propuesta de aplicación metodológica del diseño}

Para la aplicación de este diseño curricular, se propone la siguiente orientación metodológica.

\section{Recomendaciones:}

- Respetar y cumplir con los contenidos que se plantean.

- Trabajar por el logro de los objetivos propuestos. 
- Estimular la lectura comprensiva en el aula, y la recreativa en tiempo extra clase.

- Trabajar el vocabulario y uso del diccionario.

- Propiciar el análisis de obras y géneros literarios.

- Estimular la producción literaria.
- Debatir y dialogar sobre la vida y obra de los diferentes autores.

- Cumplimiento de la carga horaria semanal (225 minutos) con una frecuencia de 45 a 90 minutos diarios.

- Cantidad máxima de 15 estudiantes por aula.
Metodología
- Definir el problema
- Identificar el objeto de estudio que corresponde a la unidad temática.
- Determinar el objetivo, aspiración o propósito que dé solución al problema.

Desarrollar los contenidos de cada unidad temática

Se utilizará la metodología del Proceso Enseñanza Aprendizaje (6 etapas) (Álvarez, 2016, p.73):

- Motivación

- Información del contenido

- Asimilación del contenido

- Dominio del contenido

- Sistematización del contenido

- Evaluación del aprendizaje.

\begin{abstract}
Etapas del proceso de enseñanza aprendizaje

Trabajo independiente
\end{abstract}

Medios de enseñanza
Se sugieren los siguientes métodos:(Álvarez, 3ra Edición 2016. p.127)

- Expositivo

- Elaboración conjunta

Entre los procedimientos de enseñanza aprendizaje, se plantean los siguientes:

- Inducción y deducción

- Análisis y síntesis

- Abstracción y concentración

- Gráficos: manuales, libros de consulta, prensa escrita, guías didácticas.

- Visuales: dibujos, fotografías, pinturas, gráficos, tablas.

- Orales: Data, CD, discos, emisiones radiofónicas.

- Audiovisuales: montaje de diapositivas, películas, video, programa de televisión.

\section{Sistemáticas}

- Trabajos prácticos

- Exposiciones.

- Tareas

- Investigaciones

- Participación en clases

\section{Pruebas}

- Final de unidad temática

- Trimestral

- Diagnóstica 
La atención personalizada en la aplicación de esta metodología es la siguiente:

- Atención a las diferencias individuales.

- Actividades y tareas de acuerdo con el grado de asimilación del alumno.

- Reforzar los contenidos de mayor grado de complejidad.

- Sustentar los conocimientos y el desarrollo de habilidades y capacidades sobre la base de las relaciones afectivo- comunicativas.

- Estimular la elevación de la autoestima.

- Mantener el control y evaluación según el grado de asimilación del estudiante.

- Estimular los logros de los alumnos sistemáticamente.

- Clases dinámicas y motivadas.

- Dar tareas diferenciadas al alumno y posibilitar su participación.

- Realizar adaptación curricular para darle atención a todos los estudiantes.

- Trabajar las necesidades educativas, sentimientos y valores.

- osibilitar el cumplimiento de los objetivos de acuerdo al grado de asimilación del estudiante.

- ontrolar el rendimiento académico de los estudiantes sistemáticamente.

\section{Conclusiones de la propuesta}

El diseño curricular propuesto tiene entre sus contenidos por cursos los siguientes:

- 9no de secundaria (9) - Historia de la literatura boliviana

- 10mo de secundaria (10) - Historia de la literatura universal

- 11no de secundaria (11) - Manifestaciones del pensamiento literario latinoamericano

- 12vo de secundaria (12) - Literatura contemporánea

\section{CONCLUSIONES}

El aporte científico es producto del nuevo Diseño curricular de la asignatura Lenguaje y comunicación, con una intención personalizada que se constituye como una necesidad para los estudiantes del nivel secundario en la ciudad de Cochabamba, ya que a través de él, los estudiantes van a tener la posibilidad de concluir sus estudios secundarios con una visión de los aspectos más significativos de la historia de la literatura, el joven estará apto para relacionarse en cualquier esfera de la vida social y cultural, porque ha recibido una enseñanza individualizada, personalizada que también tributará a mejorar el rendimiento académico, teniendo en cuenta las siguientes consideraciones:

Los grupos deben conformarse por 15 alumnos como máximo, para recibir atención individualizada por parte del profesor, de forma tal que se estimule el razonamiento, la creatividad y el desarrollo de los conocimientos de la literatura universal y latinoamericana, conscientes del mundo que le rodea y el papel que le corresponde, socialmente útil, con alto poder de decisión y conocimiento amplio de cada una de las áreas, como es Lenguaje y comunicación que ayuda a la formación de un hombre apto para vivir en sociedad con capacidades necesarias para continuar estudios superiores.

Para lograr lo anteriormente expuesto, el diseño está basado en la Reforma Educativa Boliviana, el Proyecto de Ley 070 Elizardo Pérez y Avelino Siñani, Normas Internacionales para la enseñanza en el nivel secundario, experiencias metodológicas de los docentes y experiencia personal de la autora con 37 años como docente.

Por ello, se puede concluir que el diseño curricular humanista y personalizado permitirá al estudiante de nivel secundario elevar su rendimiento académico porque se toma en 
cuenta su individualidad, singularidad, autonomía y su dimensión social para la formación integral de su personalidad, es decir, en sus habilidades, capacidades, y competencias, así como valores, actitudes, carácter y convicciones a través de las actividades escolares y extraescolares y que tiene como característica la flexibilidad, pertinencia, unidad y coherencia.

Además, se evidenció que el rendimiento académico de los estudiantes de nivel secundario de Cochabamba es bajo y en ello incide el necesario dominio de la ortografía, la redacción, interpretación de textos orales y escritos, la selección acertada de textos que representen la literatura universal $\mathrm{y}$ latinoamericana. Por todo lo expuesto deben reconsiderarse los contenidos que se declaran en el diseño curricular, estableciendo relaciones sobre la base de los métodos dialéctico, holístico, sistémico y holográfico, es decir complejo, lo que determina la propuesta de un nuevo diseño referido al área de Lenguaje y Comunicación.

\section{REFERENCIAS}

Acosta, F (2012). La Escuela Secundaria Argentinaen perspectiva histórica comparada:Modelos institucionlesy desgranamiento durante el siglo XX. Cadernos de história de educación. Obtenido de SCRIBD: https://es.scribd.com/document/35991126 8/Acosta-2012-La-Escuela-SecundariaArgentina-en-Perspectiva-HistoricaModelos-Institucionales-y-DesgranamientoDurante-El-Siglo-XX

Álvarez de Zayas, C. M. (2019). Diseño Curricular. Bolivia: Kipus.

Álvarez de Zayas, C. M. (2016). Metodologia de la investigacion cientifica. Cochabamba: Kipus.

Álvarez de Zayas, R. M. (2016). Metodología de enseñanza aprendizaje. Cochabamba: Kipus

Figueroa, C. (2004). Sistemas de evaluación académica. El Salvador: Editorial Universitaria.

Gómez, A. C. (2000) Título, Lenguaje y Comunicación. Pie de Imprenta, Caracas, Venezuela

Ley de Reforma Educativa 070. (2012). Bolivia. Gaceta Oficial. ww.gacetaoficialdebolivia.gob.bo

Ministerio de Educación, República de Chile Alameda 1371, (Santiago diciembre de 2009) Marco de objetivos fundamentales y contenidos minimos obligatorios de educacion basica y media. Santiago,Chile: Actualizacion

Soacha, J. (2001). Origen de la educación personalizada. Colombia. http://www.sappiens.com/castellano/articul os.nsf/Formaci\%C3\%B3n_Virtual/Origen_de _la_educaci\%C3\%B3n_personalizada/EAC81 443CD6FC26741256AB9004007C0!opendoc ument 